\title{
Excitation spectra of a quantum ring embedded in a photon cavity
}

\author{
Thorsten Arnold, ${ }^{1}$ Chi-Shung Tang, ${ }^{2}$ Andrei Manolescu, ${ }^{3}$ and Vidar Gudmundsson ${ }^{1}$ \\ ${ }^{1}$ Science Institute, University of Iceland, Dunhaga 3, IS-107 Reykjavik, Iceland \\ ${ }^{2}$ Department of Mechanical Engineering, National United University, 1, Lienda, Miaoli 36003, Taiwan \\ ${ }^{3}$ School of Science and Engineering, Reykjavik University, Menntavegur 1, IS-101 Reykjavik, Iceland
}

\begin{abstract}
We explore the response of a quantum ring system coupled to a photon cavity with a single mode when excited by a classical dipole field. We find that the energy oscillates between the electronic and photonic components of the system. The contribution of the linear and the quadratic terms in the vector potential to the electron-photon interaction energy are of similar magnitude, but opposite signs stressing the importance of retaining both in the model. Furthermore, we find different Fourier spectra for the oscillations of the center of charge and the oscillations of the mean photon number in time. The Fourier spectra are compared to the spectrum of the many-body states and selection rules discussed. In case of the center of charge oscillations, the dipole matrix elements preselect the allowed Bohr frequencies of the transitions, while for the oscillations of the mean photon number, the difference of the photon content of the many-body states influences the selection rules.
\end{abstract}

PACS numbers: $42.50 . \mathrm{Hz}, 73.21 .-\mathrm{b}, 78.67 .-\mathrm{n}$

\section{INTRODUCTION}

Charge oscillations in mesoscopic electronic systems have been investigated for a long time by far-infrared spectroscopy $[1,2]$. Even for simple systems like quantum dots such oscillations are usually complex, incorporating the interaction of electrons with the confinement potentials and the many-body (MB) electron-electron interactions as well. Specific symmetries or selection rules may sometimes inhibit complex degrees of freedom and reduce the oscillations to the motion of the center of mass $[3,4]$. Resonant Raman scattering has also been used to observe and distinguish single-electron excitations and collective modes with monopole, dipole, or quadrupole symmetry characterized by angular momenta quantum numbers $m=0, \pm 1, \pm 2[5,6]$.

Charge oscillations, or other time-dependent phenomena in a mesoscopic system, can be induced by a radiation field $[7,8]$. It was shown theoretically that a short pulse can induce a charge current $[9,10]$ or a spin current $[11,12]$ in a ring. Given the inhomogeneous charge distribution in a nanoscale system, nonlinear response of the charge or currents to an external electromagnetic pulse is to be expected [13].

Complementary to charge or current oscillations, the interaction of electrons with photons has been at the core of condensed matter physics research for a long time. Electronic systems on the nanoscale offer a unique opportunity to study this interaction, and the possibility to control optical response. For example the dependence of optical transitions in nanostructures on shape [14, 15] and temperature [16] has been studied. In some cases, quantum rings have been preferred over quantum dots when selecting a quantum confinement since the oscillator strength for the excitonic ground state is larger [17]. The photoluminescence and the excitation spectrum of quantum rings and their optical transitions and selection rules have been investigated $[18,19]$. However, it is found that field effect structure devices reduce the oscil- lator strength [20].

The electron-photon interaction may be to some extent controlled inside optical microcavities, and such experiments on single photon emission in quantum rings have been carried out [21]. Also, photoluminescence measurements on quantum rings and optical emission of quantum dots coupled to photonic microcavities have been performed [22, 23]. Electron-photon systems in cavities, which are in fact quantum electrodynamical systems at mesoscopic scale, constitute an emerging topic of active research. Either driven by the need of elements for quantum computing [24], or due to the possibility of polariton condensation at high temperatures [25], or polariton blocking [26], or in the context of photo-assisted transport through a nanoscale system [27]. Recently, it has been shown that the charge and spin currents in quantum rings can be tuned by the interaction of electrons with photons carrying linear or circular polarization [2830].

The emission spectrum of an Aharonov-Bohm [31] quantum ring in a single mode microcavity was theoretically studied in the strong coupling regime under the influence of time-independent external fields [32]. The effect of a short light pulse on the emission of dipolaritons in quantum wells embedded in a microcavity has been discussed [33]. Oscillations of the electric dipole moment for a single electron Aharonov-Bohm ring have been related to the selection rules of the optical transitions in the ring and have been shown to allow a control of the polarization properties [34].

To the best of our knowledge, the effect of a short time-dependent electromagnetic pulse on a quantum ring coupled to a photon cavity has not been investigated. In this paper, we study numerically the time evolution of the energies of the MB electron-photon system. We calculate the dipole moment of the charge density and the mean photon number beyond two level models and the rotating wave approximation $[35,36]$. The time evolution can be considered as the non-linear response to a classical 
excitation pulse. The time evolution is analyzed using Fast Fourier transform and the selection rules between the MB states discussed. Sec. II describes the quantum ring model coupled to a photon cavity and the excitation pulse. The electron-photon interaction is described using the method of "exact" diagonalization. Sec. III presents the results and a summary is given in Sec. IV.

\section{THEORY AND MODEL}

Here, we describe the Hamiltonian of the MB electronphoton system including the potential used to model the finite width quantum ring and its time evolution during and after excitation by a short pulse.

\section{A. Many-body system Hamiltonian}

The MB Hamiltonian of the system in second quantization without the time-dependent excitation is

$$
\begin{aligned}
\hat{H}_{\mathrm{MB}}= & \int d^{2} r \hat{\mathbf{\Psi}}^{\dagger}(\mathbf{r})\left[\frac{\hat{\mathbf{p}}^{2}}{2 m^{*}}+V(\mathbf{r})\right] \hat{\mathbf{\Psi}}(\mathbf{r}) \\
& +\hbar \omega \hat{a}^{\dagger} \hat{a},
\end{aligned}
$$

with the spinor

$$
\hat{\Psi}(\mathbf{r})=\left(\begin{array}{c}
\hat{\Psi}(\uparrow, \mathbf{r}) \\
\hat{\Psi}(\downarrow, \mathbf{r})
\end{array}\right)
$$

and

$$
\hat{\mathbf{\Psi}}^{\dagger}(\mathbf{r})=\left(\hat{\Psi}^{\dagger}(\uparrow, \mathbf{r}), \hat{\Psi}^{\dagger}(\downarrow, \mathbf{r})\right)
$$

where

$$
\hat{\Psi}(x)=\sum_{k} \psi_{k}^{S}(x) \hat{C}_{k}
$$

is the field operator with $x \equiv(\mathbf{r}, \sigma), \sigma \in\{\uparrow, \downarrow\}$ and the annihilation operator, $\hat{C}_{k}$, for the single-electron state (SES) $\left|\psi_{k}^{S}\right\rangle$ in the central system. The SES $\left|\psi_{k}^{S}\right\rangle$ is the eigenstate labeled by $k$ of the Hamiltonian $\hat{H}_{S}-\hbar \omega \hat{a}^{\dagger} \hat{a}$ when we set the photonic part of the vector potential $\hat{\mathbf{A}}^{\mathrm{ph}}(\mathbf{r})$ in the momentum operator,

$$
\hat{\mathbf{p}}(\mathbf{r})=\left(\begin{array}{c}
\hat{p}_{x}(\mathbf{r}) \\
\hat{p}_{y}(\mathbf{r})
\end{array}\right)=-i \hbar \nabla+\frac{e}{c} \hat{\mathbf{A}}^{\mathrm{ph}}(\mathbf{r}),
$$

to zero.

The last term in Eq. (1) indicates the quantized photon field, where $\hat{a}^{\dagger}$ is the photon creation operator and $\hbar \omega$ is the photon excitation energy. The zero point energy, which has no other implications than a constant shift of the energy spectrum, is neglected here. The photon field interacts with the electron system via the vector potential

$$
\hat{\mathbf{A}}^{\mathrm{ph}}=A\left(\mathbf{e} \hat{a}+\mathbf{e}^{*} \hat{a}^{\dagger}\right)
$$

with

$$
\mathbf{e}=\left\{\begin{array}{cl}
\mathbf{e}_{x}, & \mathrm{TE}_{011} \\
\mathbf{e}_{y}, & \mathrm{TE}_{101} \\
\frac{1}{\sqrt{2}}\left[\mathbf{e}_{x}+i \mathbf{e}_{y}\right], & \text { RH circular } \\
\frac{1}{\sqrt{2}}\left[\mathbf{e}_{x}-i \mathbf{e}_{y}\right], & \text { LH circular }
\end{array}\right.
$$

for a longitudinally-polarized ( $x$-polarized) photon field $\left(\mathrm{TE}_{011}\right)$, transversely-polarized ( $y$-polarized) photon field $\left(\mathrm{TE}_{101}\right)$, right-hand $(\mathrm{RH})$ or left-hand $(\mathrm{LH})$ circularly polarized photon field. For reasons of simplicity, we show here only the results for the $x$-polarized photon field, but comment on the results for photon fields with other polarization. In particular, the number of allowed transitions between MB states is much larger for circular polarization. The electron-photon coupling constant

$$
g_{\gamma}=e A a_{w} \Omega_{w} / c
$$

scales with the amplitude $A$ of the electromagnetic field, and the natural length scale due to the confinement of the system,

$$
a_{w}=\left(\frac{\hbar}{m^{*} \Omega_{0}}\right)^{1 / 2}
$$

Our model of a photon cavity can be realized experimentally [37-39] by letting the photon cavity be much larger than the quantum ring (this assumption is used in the derivation of the vector potential, Eq. (6)).

\section{B. Quantum ring potential}

We model a small quantum ring with a finite width. The quantum ring confinement potential is shown in Fig.

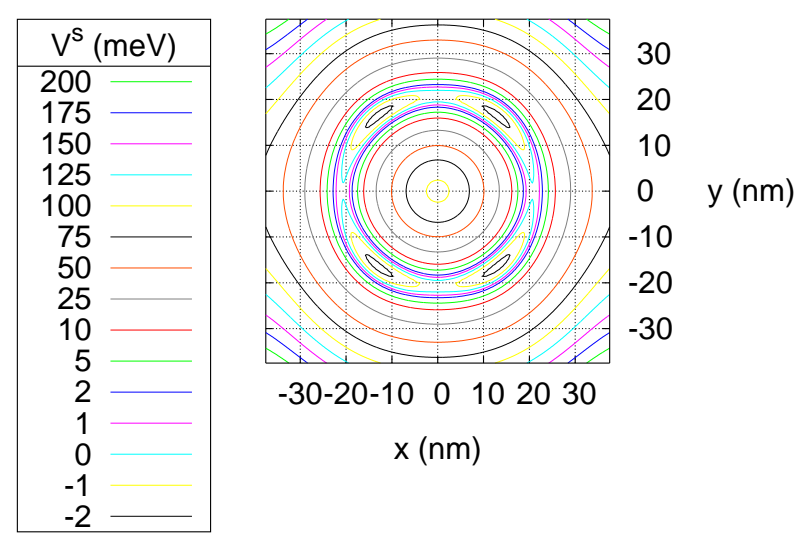

FIG. 1. (Color online) Equipotential lines of the confinement potential $V(\mathbf{r})$ of the quantum ring. The equipotential lines are refined at the bottom of the ring.

1. The ring is relatively small and the electrons relatively strongly confined to minimize the computational effort. 
TABLE I. Parameters of the ring potential $V(\mathbf{r})$.

\begin{tabular}{c|c|c|c|c}
\hline \hline & $V_{i}$ in $\mathrm{meV}$ & $\beta_{x i}$ in $\frac{1}{\mathrm{~nm}}$ & $x_{0 i}$ in $\mathrm{nm}$ & $\beta_{y i}$ in $\frac{1}{\mathrm{~nm}}$ \\
\hline 1 & 164.8 & 0.044 & 45 & 0 \\
2 & 164.8 & 0.044 & -45 & 0 \\
3 & 177.6 & 0.066 & $\epsilon$ & 0.068 \\
6 & -80.0 & 0 & 0 & 0 \\
\hline \hline
\end{tabular}

Mathematically, the expression for the potential is

$$
\begin{aligned}
V(\mathbf{r})= & \sum_{i=1}^{4} V_{i} \exp \left[-\left(\beta_{x i}\left(x-x_{0 i}\right)\right)^{2}-\left(\beta_{y i} y\right)^{2}\right] \\
& +\frac{1}{2} m^{*} \Omega_{0}^{2} y^{2},
\end{aligned}
$$

with the parameters from Tab. I and the characteristic confinement energy in $y$-direction being $\hbar \Omega_{0}=16.0 \mathrm{meV}$. $x_{03}=\epsilon$ is a small numerical symmetry breaking parameter and $|\epsilon|=10^{-5} \mathrm{~nm}$ is enough for numerical stability.

\section{Excitation pulse and time evolution}

The time evolution is given by the Liouville-von Neumann equation

$$
i \hbar \frac{d}{d t} \hat{\rho}(t)=\left[\hat{H}_{\mathrm{MB}}+\hat{W}_{\mathrm{MB}}(t), \hat{\rho}(t)\right]
$$

with the MB operator $\hat{W}_{\mathrm{MB}}(t)$ representing a short excitation pulse

$$
\begin{aligned}
W(\mathbf{r}, t)= & W_{d}(\mathbf{r}) \exp (-\Gamma t) \\
& \times \sin \left(\omega_{2} t\right) \sin \left(\omega_{1} t\right) \theta\left(10 \pi-\omega_{2} t\right)
\end{aligned}
$$

with the dipole potential

$$
W_{d}(\mathbf{r})=W_{0} x
$$

in $x$-direction with $W_{0}=2.36 \times 10^{-2} \mathrm{meV} / \mathrm{nm}$.

In Eq. (11), the density operator $\hat{\rho}(t)$ and the other operators appear in the MB presentation. Numerically, we solve the Liouville-von Neumann equation Eq. (11) using the time-evolution operator $\hat{U}_{\mathrm{MB}}(t)$ defined by [9]

$$
\hat{\rho}(t)=\hat{U}_{\mathrm{MB}}(t) \hat{\rho}(0) \hat{U}_{\mathrm{MB}}^{\dagger}(t)
$$

yielding the equations of motion

$$
\begin{aligned}
i \hbar \dot{\hat{U}}_{\mathrm{MB}}(t) & =\hat{H}_{\mathrm{MB}}^{\mathrm{td}}(t) \hat{U}_{\mathrm{MB}}(t), \\
-i \hbar \dot{\hat{U}}_{\mathrm{MB}}^{\dagger}(t) & =\hat{U}_{\mathrm{MB}}^{\dagger}(t) \hat{H}_{\mathrm{MB}}^{\mathrm{td}}(t)
\end{aligned}
$$

with the time-dependent MB Hamiltonian

$$
\hat{H}_{\mathrm{MB}}^{\mathrm{td}}(t)=\hat{H}_{\mathrm{MB}}+\hat{W}_{\mathrm{MB}}(t) .
$$

The time integration of Eq. (15) is done using the CrankNicolson algorithm with the initial condition $\hat{U}_{\mathrm{MB}}(0)=\mathbb{1}$ and with $\hat{H}_{\mathrm{MB}}^{\mathrm{td}}(0)=\hat{H}_{\mathrm{MB}}[9]$.

\section{RESULTS}

For all our results, we start the system in the ground state with a photon content close to zero and propagate the system long after the external pulse has vanished, until the time $t=440$ ps to get a precise description of the Fourier transform (FT) of various oscillations the system is performing. We assume a GaAs-based material with the electron effective mass $m^{*}=0.067 m_{e}$ and background relative dielectric constant $\kappa=12.4$. The single photon cavity mode has the excitation energy $\hbar \omega=6.4 \mathrm{meV}$ and the energies of the excitation pulse $\hbar \omega_{1}=2.63 \mathrm{meV}$ and $\hbar \omega_{2}=0.658 \mathrm{meV}$ and the decay factor $\Gamma=0.2 \mathrm{ps}^{-1}$. A magnetic field $B=10^{-5} \mathrm{~T}$ five orders of magnitude below the Aharonov-Bohm [31] regime for our ring size is used here for the numerical purpose to lift the spin degeneracy. The length scale (defined in Eq. (9)) is $a_{w} \approx 8.431 \mathrm{~nm}$.

The electron number in the system is fixed to one electron to reduce the numerical effort and to focus the attention on the effects of the electron-photon interaction. Therefore, the Coulomb interaction does not have to be considered in Eq. (1).

\section{A. Energetics analysis of the system}

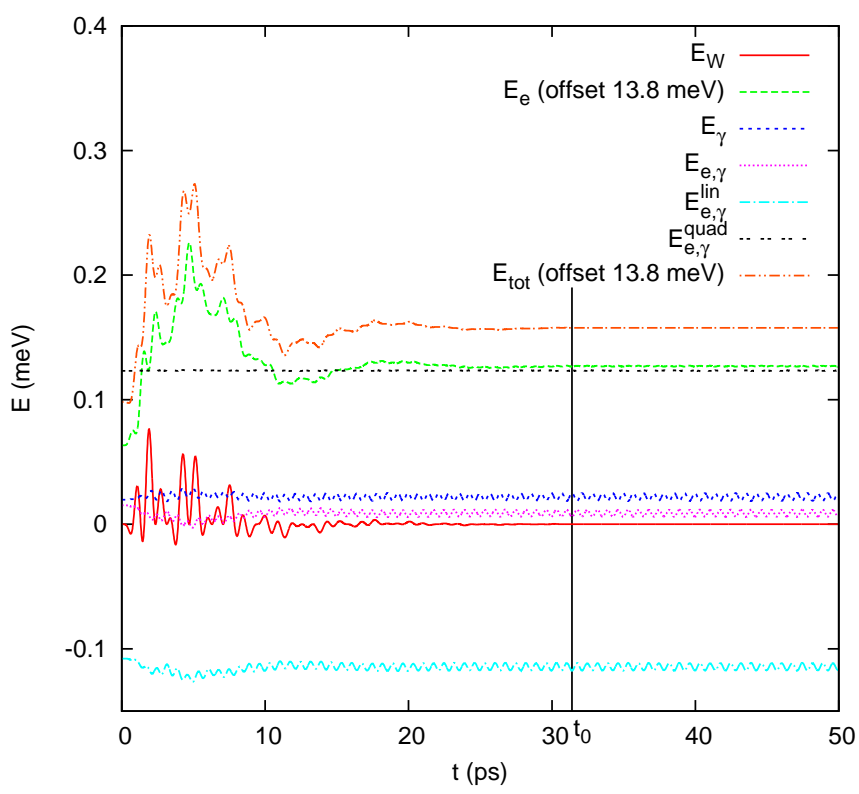

FIG. 2. (Color online) Energies in the system as a function of time. The zero point energy of the photons is excluded. Two very positive energy curves have been reduced by a convenient offset of $13.8 \mathrm{meV}$ to smaller values to fall in a similar energy range as the other curves. The photon field is $x$-polarized. The electron-photon coupling constant $g_{\gamma}=0.5 \mathrm{meV}$. The end of the excitation pulse is marked by a vertical line at $t=$ $t_{0}:=10 \pi / \omega_{2}=31.4$ ps. Energies are defined by Equations (17)-(25). 
The quantum ring system is filled with an electron coupled to cavity photons of a single frequency and is influenced by a weak dipole excitation pulse in the $x$-direction. To understand the time-dependency of the different components of the system, we take first a look at their energy contents in Fig. 2. The total energy (without zero point energy of the photons)

$$
E_{\text {tot }}(t)=\operatorname{Tr}\left[\hat{\rho}(t) \hat{H}_{\mathrm{MB}}^{\mathrm{td}}(t)\right]
$$

is a constant after the excitation pulse $\left(t>t_{0}:=\right.$ $10 \pi / \omega_{2}=31.4 \mathrm{ps}$ ), as the system can then be considered to be closed relative to its environment. However, the MB system energy can be redistributed and oscillate between the electron and the photons after the excitation by the pulse. More correctly, there are three energy contributions after the pulse, the energy of the electron

$$
E_{e}(t)=\operatorname{Tr}\left[\hat{\rho}(t) \hat{H}_{\mathrm{MB}}^{e}\right]
$$

with

$$
\hat{H}_{\mathrm{MB}}^{e}=\int d^{2} r \hat{\boldsymbol{\Psi}}^{\dagger}(\mathbf{r})\left[\frac{\hat{\mathbf{p}}^{\prime 2}}{2 m^{*}}+V(\mathbf{r})\right] \hat{\mathbf{\Psi}}(\mathbf{r})
$$

and

$$
\hat{\mathbf{p}}^{\prime}=-i \hbar \nabla
$$

the energy of the photons (without zero point energy)

$$
E_{\gamma}(t)=\operatorname{Tr}\left[\hat{\rho}(t) \hbar \omega \hat{a}^{\dagger} \hat{a}\right]
$$

and the energy due to the interaction term of the electron and the photons

$$
E_{e, \gamma}(t)=\operatorname{Tr}\left[\hat{\rho}(t) \hat{H}_{\mathrm{MB}}^{e, \gamma}\right]
$$

with

$$
\begin{gathered}
\hat{H}_{\mathrm{MB}}^{e, \gamma}=\hat{H}_{\mathrm{MB}, \text { lin }}^{e, \gamma}+\hat{H}_{\mathrm{MB}, \mathrm{par}}^{e, \gamma} \\
\hat{H}_{\mathrm{MB}, \operatorname{lin}}^{e, \gamma}=\int d^{2} r \hat{\mathbf{\Psi}}^{\dagger}(\mathbf{r})\left[\frac{\hat{\mathbf{p}}^{\prime} \hat{\mathbf{A}}^{\mathrm{ph}}(\mathbf{r})+\hat{\mathbf{A}}^{\mathrm{ph}}(\mathbf{r}) \hat{\mathbf{p}}^{\prime}}{2 m^{*} c / e}\right] \hat{\mathbf{\Psi}}(\mathbf{r})
\end{gathered}
$$

and

$$
\hat{H}_{\mathrm{MB}, \mathrm{par}}^{e, \gamma}=\int d^{2} r \hat{\boldsymbol{\Psi}}^{\dagger}(\mathbf{r})\left[\frac{e^{2}\left(\hat{\mathbf{A}}^{\mathrm{ph}}(\mathbf{r})\right)^{2}}{2 m^{*} c^{2}}\right] \hat{\boldsymbol{\Psi}}(\mathbf{r})
$$

The electron energy is by far the largest contribution (around $13.9 \mathrm{meV}$ ), the photon energy is about $3.2 \mathrm{meV}$ when adding the zero point energy and the electronphoton interaction energy is much smaller than $0.1 \mathrm{meV}$. However, when separating the interaction energy into two terms with a linear,

$$
E_{e, \gamma}^{\operatorname{lin}}(t)=\operatorname{Tr}\left[\hat{\rho}(t) \hat{H}_{\mathrm{MB}, \operatorname{lin}}^{e, \gamma}\right]
$$

or quadratic,

$$
E_{e, \gamma}^{\mathrm{quad}}(t)=\operatorname{Tr}\left[\hat{\rho}(t) \hat{H}_{\mathrm{MB}, \mathrm{par}}^{e, \gamma}\right]
$$

dependency on the vector potential $\hat{\mathbf{A}}^{\mathrm{ph}}(\mathbf{r})$, it can be seen that each of this contribution is larger than $0.1 \mathrm{meV}$, but with opposite signs.

All energy contributions show similar oscillations after the excitation pulse and pass energy between each other. In particular, the oscillation of the linear interaction energy, Eq. (26), and photon energy, Eq. (21), are strong and almost in anti-phase. In the following, we will concentrate on the oscillations of the photon energy or more precisely said, the oscillations in the mean photon number, which is proportional to the photon energy.

During the excitation pulse, there is an additional energy appearing due to the excitation pulse itself

$$
E_{W}(t)=\operatorname{Tr}\left[\hat{\rho}(t) \hat{W}_{\mathrm{MB}}(t)\right]
$$

The excitation pulse can also increase or decrease the total energy of the otherwise closed system. The change of the total energy is relatively small compared to the photon excitation energy $\hbar \omega$. In this sense, the excitation pulse can be considered to be weak meaning that it can not change the mean photon number by a whole photon.

\section{B. Dipole moment oscillations of the charge density}

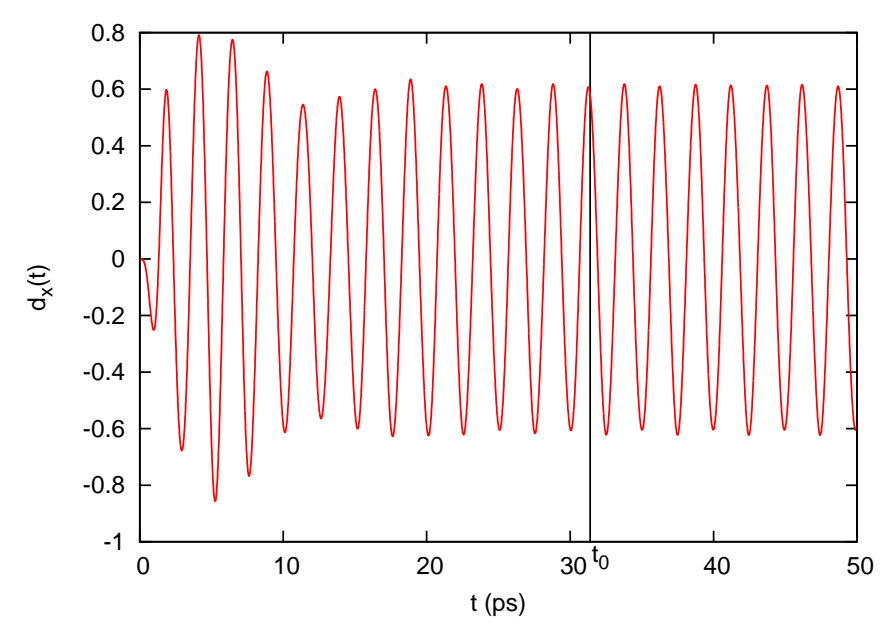

FIG. 3. (Color online) Dipole moment oscillations of the charge density distribution in $x$-direction as a function of time. The photon field is $x$-polarized. The electron-photon coupling constant $g_{\gamma}=0.5 \mathrm{meV}$. The end of the excitation pulse is marked by a vertical line at $t=t_{0}$.

It is interesting to investigate the response of the charge density to the external excitation of the quantum ring system. Excited by the dipole excitation pulse in $x$-direction, the center of charge (dipole moment of the 
charge density)

$$
\mathbf{d}(t)=\operatorname{Tr}[\hat{\rho}(t) \hat{\mathbf{d}}]
$$

with $\hat{\mathbf{d}}$ being the dipole operator,

$$
\hat{d}_{i}=e \int d^{2} r r_{i} \hat{\boldsymbol{\Psi}}^{\dagger}(\mathbf{r}) \hat{\boldsymbol{\Psi}}(\mathbf{r})
$$

where $i=x, y$, oscillates as is shown in Fig. 3. The oscillations are a superposition of periodic oscillations after the excitation pulse, but not during the time, the pulse is exciting the system. We therefore exclude the excitation time interval $t<t_{0}$ for a further analysis of the oscillations.

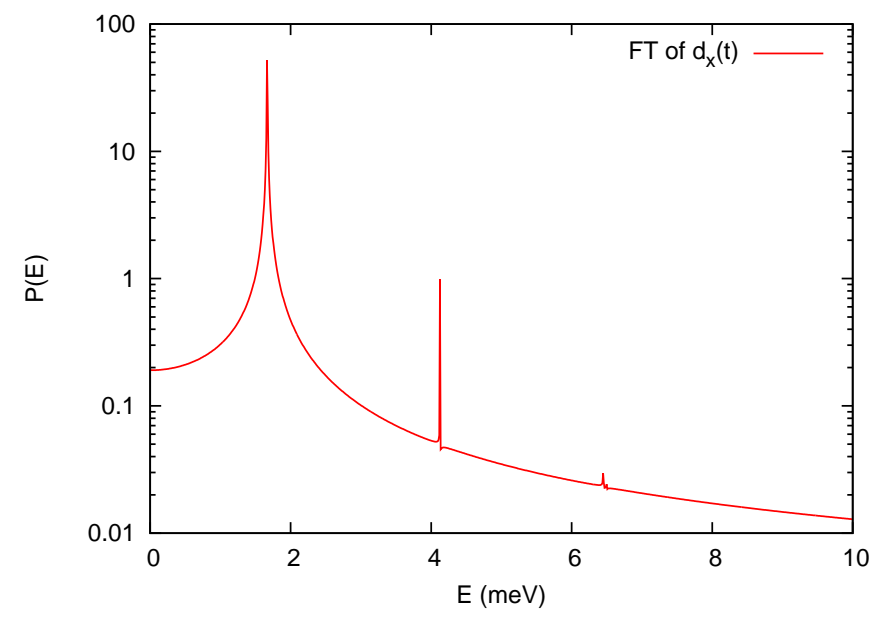

FIG. 4. (Color online) Logarithmic plot of the FT of the dipole moment oscillations of the charge density distribution as a function of energy after the excitation pulse $\left(t>t_{0}\right)$. The photon field is $x$-polarized. The electron-photon coupling constant $g_{\gamma}=0.5 \mathrm{meV}$.

Figure 4 shows the FT of the center of charge oscillations. It is mainly composed of two peaks, a strong one at $E=1.66 \mathrm{meV}$ and a much weaker one at $E=4.13 \mathrm{meV}$. (Notice the logarithmic scale of the peak height.) For $x$ - or $y$-polarized (linearly polarized) cavity photon field the center of charge oscillates in $x$-direction, meaning that $d_{y}(t)$ is vanishing. We have seen, however, that a circularly polarized cavity photon field leads to center of charge oscillations in both $d_{x}(t)$ and $d_{y}(t)$, even though the excitation pulse is only a dipole excitation in $x$-direction. Alternatively, a strong magnetic field leads also to center of charge oscillations in both the $x$ - and $y$ direction, even though the cavity photon field is linearly polarized.

\section{Fourier analysis of the mean photon number oscillations}

We have seen earlier that the photon energy oscillates also in time. In Fig. 5, we show the related mean photon

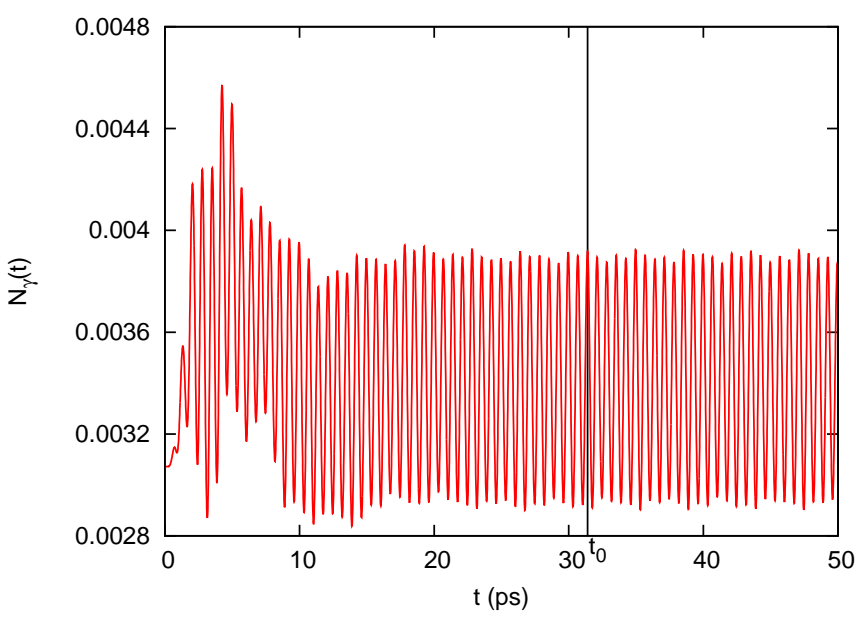

FIG. 5. (Color online) Mean photon number oscillations as a function of time. The photon field is $x$-polarized. The electron-photon coupling constant $g_{\gamma}=0.5 \mathrm{meV}$. The end of the excitation pulse is marked by a vertical line at $t=t_{0}$.

number

$$
\left\langle N_{\gamma}(t)\right\rangle=\operatorname{Tr}\left[\hat{\rho}(t) \hat{a}^{\dagger} \hat{a}\right]
$$

as a function of time, which describes the number of cavity photons in the system. Similar to Fig. 3, the oscillations are a superposition of periodic oscillations after the excitation pulse, but the main components are of a higher frequency.

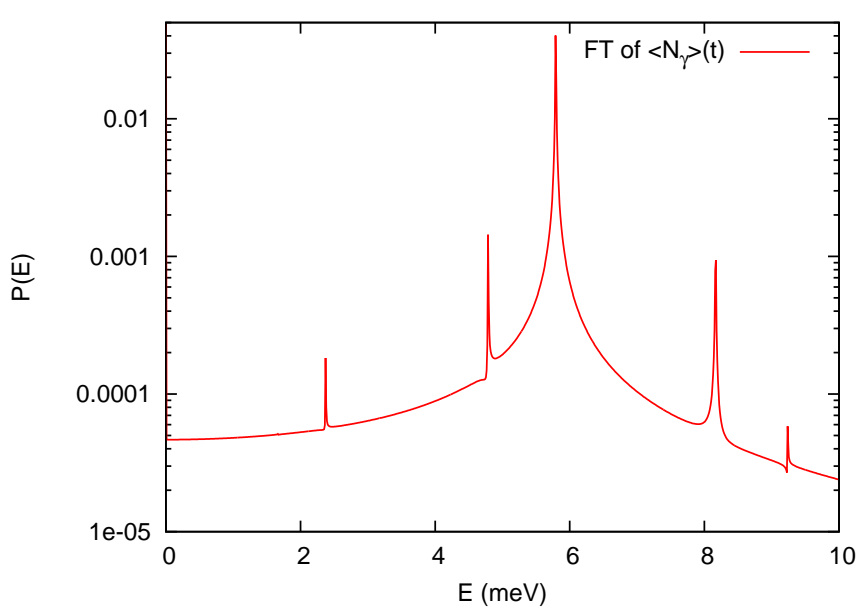

FIG. 6. (Color online) Logarithmic plot of the FT of the mean photon number oscillations as a function of energy after the excitation pulse $\left(t>t_{0}\right)$. The photon field is $x$-polarized. The electron-photon coupling constant $g_{\gamma}=0.5 \mathrm{meV}$.

This can be seen more clearly from the Fourier analysis in Fig. 6, which shows the main peaks at the energies $E=2.37 \mathrm{meV}, E=4.79 \mathrm{meV}, E=5.79 \mathrm{meV}$ and $E=8.17 \mathrm{meV}$, which would all be visible in a linear plot with the largest Fourier peak at the relatively high energy $E=5.79 \mathrm{meV}$. As mentioned earlier the oscillations 
of the electron energy and electron-photon interaction energy are composed of a similar frequency spectrum as the mean photon number. But why are the center of charge oscillations with a different spectral composition (Fig. 4)?

D. Comparison with the many-body spectrum

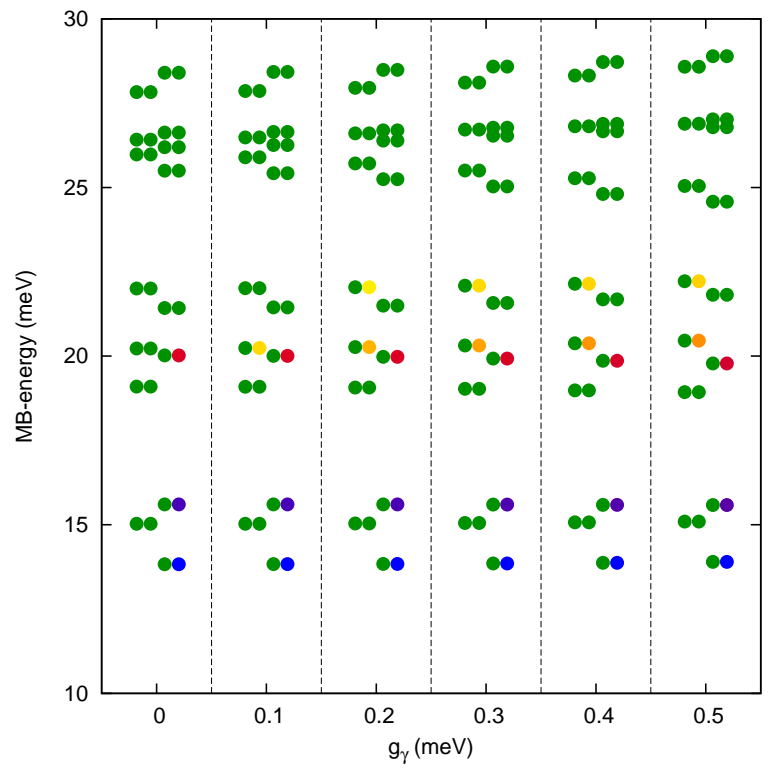

FIG. 7. (Color online) MB energy spectrum of the system Hamiltonian Eq. (1) versus the electron-photon coupling constant $g_{\gamma}$ for $x$-polarized photon field. The occupation of the states at $t=440 \mathrm{ps}$ is indicated by the color of the dots with the continuous color spectrum from yellos over red to blue corresponding to the range of the occupation number $\left[5 \times 10^{-9}, 1\right]$. If the occupation number is below $5 \times 10^{-9}$, the dots are colored green. The occupation of all MB states with an energy above $30 \mathrm{meV}$ is below $5 \times 10^{-9}$. Due to the small energy differences between MB states it was necessary to delocate the dots slightly along the $g_{\gamma}$-axis such that their occupation can be clearly recognized. This is not indicating slightly different $g_{\gamma}$-values, all dots belong exclusively to $g_{\gamma}=0,0.1,0.2,0.3,0.4,0.5$.

To understand the Fourier components of the oscillations in the center of charge and the mean photon number, we have a look at the MB energy spectrum of the quantum ring system and the occupation of the MB states at $t=440 \mathrm{ps}$ in Fig. 7. Only a few states are with an occupation above $5 \times 10^{-9}$, which we find all relevant to understand the visible peaks in a linear plot. (The sum over the occupation of all MB states is one.) This is showing that the dipole excitation pulse is not very strong, it is however strong enough that we would expect differences in the results, when we would compare to a linear response calculation. Furthermore, the relevant MB states lie all at rather low energies, hinting at the fact that the selected $N_{\mathrm{MB}}=120 \mathrm{MB}$ states are sufficient to predict the time-evolution of the system with reasonable accuracy (only the lowest MB states are shown in Fig. 7). The energetic position and occupation of the MB states changes slightly with the electron-photon coupling constant $g_{\gamma}$.

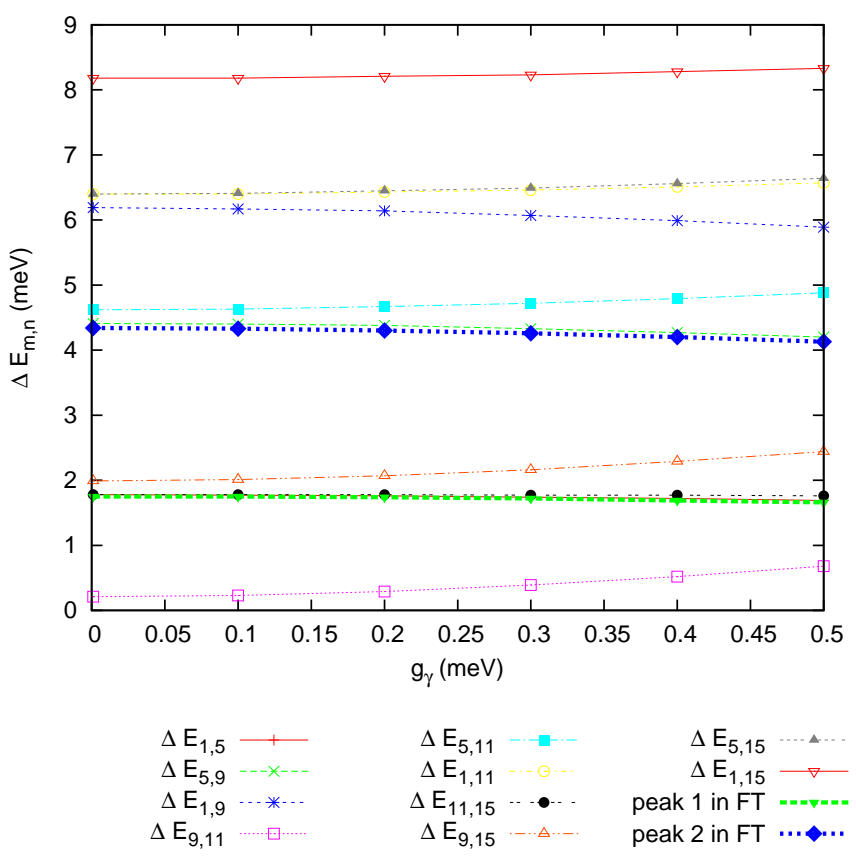

FIG. 8. (Color online) Bohr energies $\Delta E_{m, n}$ between the MB states $m$ and $n$ of the system Hamiltonian Eq. (1) and energetic location of the main peaks of the FT of the dipole moment of the charge density distribution versus the electronphoton coupling constant $g_{\gamma}$. The photon field is $x$-polarized.

To compare the MB spectrum Fig. 7 to the Fourier analysis of the center of charge oscillations, we look at Fig. 8. Here, we have plotted the Bohr energies $\Delta E_{m, n}$ between all $\mathrm{MB}$ states with occupation above $5 \times 10^{-9}$ and compare them to the peaks of the FT of the center of charge oscillations. We can see that not all transitions are likely or allowed and that some selection rules apply. In particular, we find the allowed transitions by considering the value of the matrix element

$$
W_{m, n}=\left\langle m\left|\hat{W}_{d, \mathrm{MB}}\right| n\right\rangle
$$

with $\hat{W}_{d, \mathrm{MB}}$ being the MB representation of the dipole potential Eq. (13). The Bohr energies of the allowed transitions with large $\left|W_{m, n}\right|$ are $\Delta E_{1,5}, \Delta E_{5,9}$ and $\Delta E_{11,15}$. These transitions are allowed for the whole $g_{\gamma}$-range. In addition, for $g_{\gamma}>0$, the Bohr energies $\Delta E_{9,11}, \Delta E_{1,11}$ and $\Delta E_{5,15}$ are allowed. The Bohr energies $\Delta E_{1,5}$ and $\Delta E_{5,9}$ can be recognized as peaks of the FT of the center of charge oscillations. The occupation of the higher MB states $n>9$ is very low, such that the transition $\Delta E_{11,15}$ is very weak. The same argument applies for the transitions, which are only allowed for $g_{\gamma}>0$. When 
comparing the two allowed and likely FT peaks in their strength, the peak with $\Delta E_{1,5}$ is dominant over the peak with $\Delta E_{5,9}$. This can be directly correlated to the fact that the occupation of the MB state $n=1$ is much larger than the occupation of the MB state $n=9$.
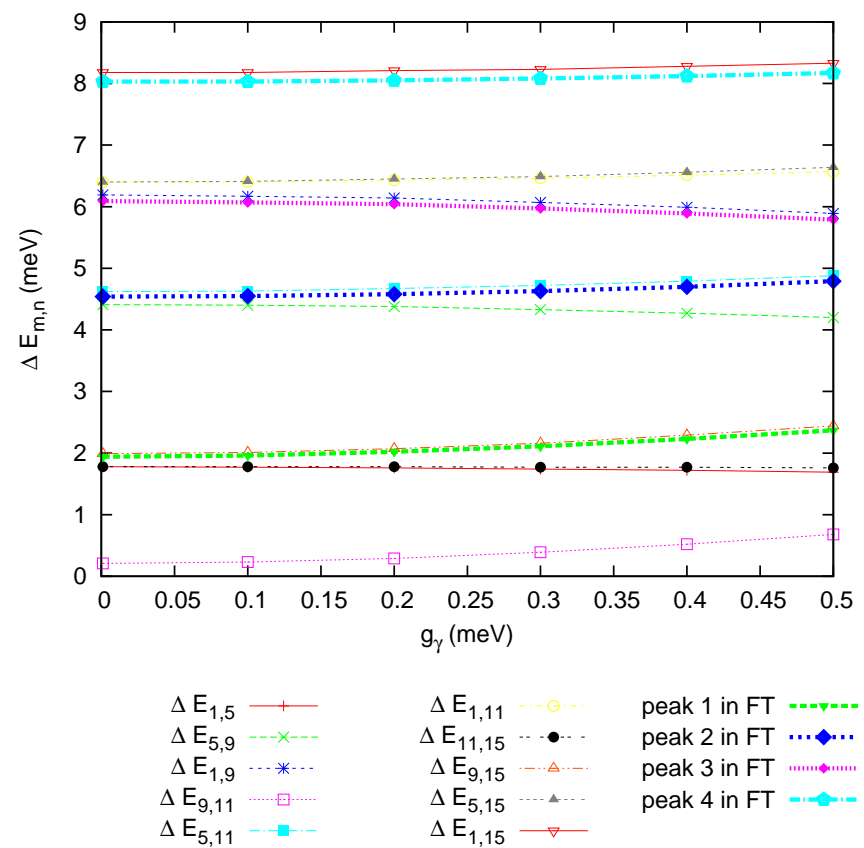

FIG. 9. (Color online) Bohr energies $\Delta E_{m, n}$ between the MB states $m$ and $n$ of the system Hamiltonian Eq. (1) and energetic location of the main peaks of the FT of the mean photon number versus the electron-photon coupling constant $g_{\gamma}$. The photon field is $x$-polarized.

Figure 9 shows the comparison of the MB spectrum Fig. 7 to the Fourier analysis of the mean photon number oscillations. We note here, that we can associate a fractional photon content $\mu$ to each MB state. The deviation of the photon content from integer numbers increases in general with the electron-photon coupling strength $g_{\gamma}$. Still, we can approximately state that the MB states $n=1,5,9$ are with a photon content close to zero and $n=11,15$ are with a photon content close to one. The transitions observed as FT peaks of the mean photon number are $\Delta E_{9,15}, \Delta E_{5,11}, \Delta E_{1,9}$ and $\Delta E_{1,15}$. Except for the strongest peak with $\Delta E_{1,9}$, the photon content is changing in all other transitions by approximately one $(\Delta \nu \approx 1)$. For the peak with $\Delta E_{1,9}$, the photon content difference of the states is $\Delta \nu=0.096$ for $g_{\gamma}=0.5 \mathrm{meV}$ and gets smaller with decreasing $g_{\gamma}$. However, it is clear from Fig. 7 that the occupation of the excited $\mathrm{MB}$ state for the transitions with $\Delta E_{9,15}$, $\Delta E_{5,11}$ and $\Delta E_{1,15}$ becomes also smaller with decreasing $g_{\gamma}$. As a consequence, we found all Fourier peaks of the mean photon number to become smaller with decreasing $g_{\gamma}$, but their relative strength is almost conserved (i.e. the main change of Fig. 6 with $g_{\gamma}$ is only the scaling of the $y$-axis). Therefore, we have to consider both the change in the photon content $\Delta \nu$ and the occupations of the two MB states associated with the transition to be able to say something about the selection rules governing the optical transitions. As a side remark, we could also associate an angular momentum $M$ to each MB state with increasing deviations from integer angular momentum numbers $m=M / \hbar$ with increasing electron-photon coupling strength $g_{\gamma}$. The absolute value of the angular momentum number $|m| \approx 0$ for the MB states $n=1,11$; $|m| \approx 1$ for the MB states $n=5,15$; and $|m| \approx 2$ for the MB state $n=9$. The MB states corresponding to the FT peaks of the mean photon number show a difference of $\Delta|m| \approx 1$ with one exception with the Bohr energy $\Delta E_{1,9}$, where $\Delta|m| \approx 2$.

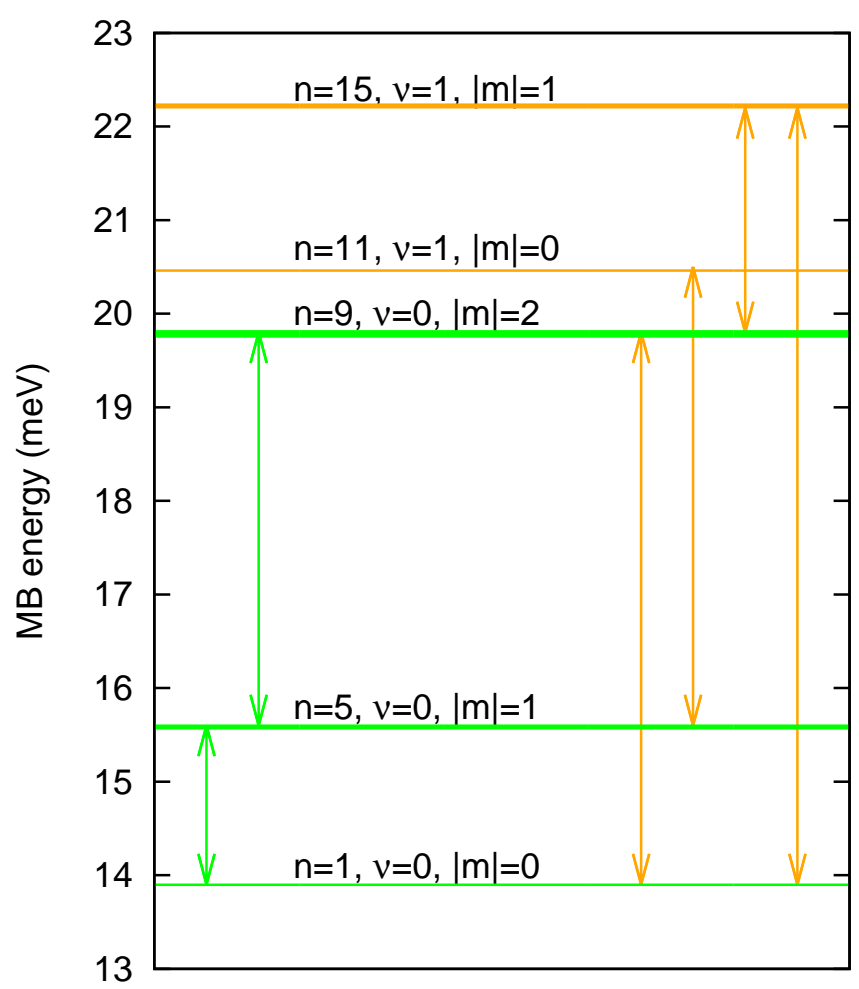

FIG. 10. (Color online) Mostly occupied MB energy levels and transitions between them. The number $n$ is the level number for energetic ordering of the MB states, $\nu$ is the approximate photon content and $|m|$ the approximate absolute value of the angular momentum number. The line width increases with $|m|$ in the cartoon and the green color means an approximate photon content $\nu \approx 0$, while the orange color means an approximate photon content $\nu \approx 1$. The green transitions correspond to the Bohr energies seen as main peaks in the FT of the center of charge oscillations and the orange transitions correspond to the Bohr energies seen as main peaks in the FT of the mean photon number oscillations. The electronphoton coupling constant $g_{\gamma}=0.5 \mathrm{meV}$. The photon field is $x$-polarized.

The approximate values of the absolute value of the angular momentum numbers $m$ and photon contents $\nu$ for the mostly populated MB levels are depicted in Fig. 
10. It shows also the Bohr energies, which describe the frequencies of the center of charge oscillations and the frequencies of the oscillations of the mean photon number. One could pose the interesting question concerning the transition with Bohr energy $\Delta E_{1,9}$ of the latter type, where $\Delta|m| \approx 2$ and $\Delta \nu \approx 0$, whether this transition would in fact be composed of two processes, each with $\Delta|m| \approx 1$ and possibly a photon content difference $\Delta \nu \approx 1$ in the first and $\Delta \nu \approx-1$ in the second process.

\section{CONCLUSIONS}

We have studied the non-linear response of a quantum ring system to a short dipole excitation pulse. The quantum ring system is coupled to a photon cavity using exact numerical diagonalization. We have seen that the many-level description is essential to describe properly the physical response of the system. The short pulse excites oscillations of the energy between the electron and cavity photons. The coupling energy between the photons and electron is small as the linear and quadratic term in the vector potential are of opposite signs. We find center of charge oscillations in the direction of the dipole excitation of the pulse. The direction of the linear polarized cavity photon field does not influence the center of charge oscillation direction. A circularly polarized cavity photon field or magnetic field, however, changes the direction. The oscillations of the mean photon number and center of charge have a different Fourier spectrum, but are all reflected in transition energies between $\mathrm{MB}$ levels. The oscillator strengths for the center of charge oscillation spectrum are given by selection rules due to the matrix elements of the dipole potential with the MB states corresponding to a MB transition (i.e. the geometrical symmetry of the MB states) and the population of the levels. For the selection rules governing the mean photon number oscillations, the difference of the photon content of the MB states determining a transition and the MB level population play a crucial role.

In summary, we have supplied a rather small amount of energy to the system with an excitation pulse with a rather broad frequency spectrum, we are thus not exciting isolated resonances in the system, but instead probing its response to a broad frequency range. It is demonstrated why both the linear and quadratic electronphoton interactions are necessary together with a large section of the system states.

\section{ACKNOWLEDGMENTS}

This work was financially supported by the Icelandic Research and Instruments Funds, the Research Fund of the University of Iceland, and the Ministry of Science and Technology, Taiwan through Contract No. MOST 103-2112-M-239-001-MY3. We acknowledge also support from the computational facilities of the Nordic High Performance Computing (NHPC).
[1] T. Demel, D. Heitmann, P. Grambow, and K. Ploog, Phys. Rev. Lett. 64, 788 (1990).

[2] S. M. Reimann and M. Manninen, Rev. Mod. Phys. 74, 1283 (2002).

[3] V. Gudmundsson and R. R. Gerhardts, Phys. Rev. B 43, 12098 (1991).

[4] D. Pfannkuche and R. R. Gerhardts, Phys. Rev. B 44, 13132 (1991).

[5] C. Steinebach, C. Schüller, and D. Heitmann, Phys. Rev. B 59, 10240 (1999).

[6] C. Steinebach, C. Schüller, and D. Heitmann, Phys. Rev. B 61, 15600 (2000).

[7] R. G. Mani, J. H. Smet, K. von Klitzing, V. Narayanamurti, W. B. Johnson, and V. Umansky, Nature (London) 420, 646 (2002).

[8] C. S. Tang and C. S. Chu, Solid State Commun. 120, 353 (2001).

[9] V. Gudmundsson, C.-S. Tang, and A. Manolescu, Phys. Rev. B 67, 161301 (2003).

[10] Z.-G. Zhu and J. Berakdar, J. Phys.: Cond. Matt. 21, 145801 (2009).

[11] M. Nita, D. C. Marinescu, A. Manolescu, and V. Gudmundsson, Phys. Rev. B 83, 155427 (2011).

[12] M. Ni, D. Marinescu, A. Manolescu, B. Ostahie, and V. Gudmundsson, Physica E 46, 12 (2012).

[13] V. Gudmundsson, S. Hauksson, A. Johnsen, G. Reinisch, A. Manolescu, C. Besse, and G. Dujardin, Annalen der
Physik 526, 235 (2014)

[14] I. Magnúsdóttir and V. Gudmundsson, Phys. Rev. B 60, 16591 (1999).

[15] J. Gomis, J. Martnez-Pastor, B. Aln, D. Granados, J. M. Garca, and P. Roussignol, Eur. Phys. J. B 54, 471 (2006).

[16] C. H. Lin, H. S. Lin, C. C. Huang, S. K. Su, S. D. Lin, K. W. Sun, C. P. Lee, Y. K. Liu, M. D. Yang, and J. L. Shen, Appl. Phys. Lett. 94, 183101 (2009).

[17] H. Pettersson, R. Warburton, A. Lorke, K. Karrai, J. Kotthaus, J. Garcia, and P. Petroff, Physica E 6, 510 (2000).

[18] B. Alén, J. Martínez-Pastor, D. Granados, and J. M. García, Phys. Rev. B 72, 155331 (2005).

[19] H. D. Kim, K. Kyhm, R. A. Taylor, G. Nogues, K. C. Je, E. H. Lee, and J. D. Song, Appl. Phys. Lett. 102, 033112 (2013).

[20] B. Alén, J. Bosch, D. Granados, J. MartínezPastor, J. M. García, and L. González, Phys. Rev. B 75, 045319 (2007).

[21] E. Gallardo, L. J. Martnez, A. K. Nowak, D. Sarkar, D. Sanvitto, H. P. van der Meulen, J. M. Calleja, I. Prieto, A. R. Alija, D. Granados, A. G. Taboada, J. M. Garca, and P. A. Postigo, J.Phys.: Conf. Ser. 210, 012037 (2010).

[22] D. Sarkar, L. Martnez, I. Prieto-Gonzlez, H. van der Meulen, J. Calleja, D. Granados, A. Taboada, J. Garca, 
A. Alija, and P. Postigo, Physica E 40, 2156 (2008).

[23] E. Gallardo, L. J. Martínez, A. K. Nowak, H. P. van der Meulen, J. M. Calleja, C. Tejedor, I. Prieto, D. Granados, A. G. Taboada, J. M. García, and P. A. Postigo, Opt. Express 18, 13301 (2010).

[24] X.-Z. Yuan, H.-S. Goan, and K.-D. Zhu, Phys. Rev. B 75, 045331 (2007).

[25] F. P. Laussy, T. Taylor, I. A. Shelykh, and A. V. Kavokin, J. Nanophoton. 6, 064502 (2012).

[26] A. Verger, C. Ciuti, and I. Carusotto, Phys. Rev. B 73, 193306 (2006).

[27] G. Platero and R. Aguado, Physics Reports 395, 1 (2004).

[28] T. Arnold, C.-S. Tang, A. Manolescu, and V. Gudmundsson, Phys. Rev. B 87, 035314 (2013).

[29] T. Arnold, C.-S. Tang, A. Manolescu, and V. Gudmundsson, Eur. Phys. J. B 87, 113 (2014).

[30] T. Arnold, C.-S. Tang, A. Manolescu, and V. Gudmundsson, Physica E 60, 170 (2014).

[31] Y. Aharonov and D. Bohm, Phys. Rev. 115, 485 (1959).
[32] A. M. Alexeev, I. A. Shelykh, and M. E. Portnoi, Phys. Rev. B 88, 085429 (2013).

[33] O. Kyriienko, A. V. Kavokin, and I. A. Shelykh, Phys. Rev. Lett. 111, 176401 (2013).

[34] A. M. Alexeev and M. E. Portnoi, Phys. Rev. B 85, 245419 (2012).

[35] M. Nakano and K. Yamaguchi, Chem. Phys. Lett. 295, 328 (1998).

[36] O. Jonasson, C.-S. Tang, H.-S. Goan, A. Manolescu, and V. Gudmundsson, New Journal of Physics 14, 013036 (2012).

[37] H. Walther, B. T. H. Varcoe, B.G. Englert, and T. Becker, Reports on Progress in Physics 69, 1325 (2006).

[38] R. Miller, T. E. Northup, K. M. Birnbaum, A. Boca, A. D. Boozer, and H. J. Kimble, J. of Phys. B: Atomic, Molecular and Optical Physics 38, S551 (2005).

[39] M. Kaniber, A. Laucht, A. Neumann, M. Bichler, M.C. Amann, and J. J. Finley, J. Phys.: Cond. Matt. 20, 454209 (2008). 